\title{
Marginal bone loss around non-submerged implants is associated with salivary microbiome during bone healing
}

\author{
Xiao-Bo Duan ${ }^{1}$, Ting-Xi Wu ${ }^{2}$, Yu-Chen Guo ${ }^{1}$, Xue-Dong Zhou ${ }^{1,3}$, Yi-Ling Lei ${ }^{4}$, Xin Xu ${ }^{1,3}$, An-Chun Mo ${ }^{1,4}$, \\ Yong-Yue Wang ${ }^{1,4}$ and Quan Yuan ${ }^{1,4}$
}

Marginal bone loss during bone healing exists around non-submerged dental implants. The aim of this study was to identify the relationship between different degrees of marginal bone loss during bone healing and the salivary microbiome. One hundred patients were recruited, and marginal bone loss around their implants was measured using cone beam computed tomography during a 3-month healing period. The patients were divided into three groups according to the severity of marginal bone loss. Saliva samples were collected from all subjected and were analysed using $16 \mathrm{~S}$ MiSeq sequencing. Although the overall structure of the microbial community was not dramatically altered, the relative abundance of several taxonomic groups noticeably changed. The abundance of species in the phyla Spirochaeta and Synergistetes increased significantly as the bone loss became more severe. Species within the genus Treponema also exhibited increased abundance, whereas Veillonella, Haemophilus and Leptotrichia exhibited reduced abundances, in groups with more bone loss. Porphyromonasgingivalis, Treponemadenticola and Streptococcus intermedius were significantly more abundant in the moderate group and/or severe group. The severity of marginal bone loss around the non-submerged implant was associated with dissimilar taxonomic compositions. An increased severity of marginal bone loss was related to increased proportions of periodontal pathogenic species. These data suggest a potential role of microbes in the progression of marginal bone loss during bone healing.

International Journal of Oral Science (2017) 9, 95-103; doi:10.1038/ijos.2017.18; published online 16 June 2017

Keywords: dental implant; illumina sequencing; marginal bone loss; oral microbiome; peri-implantitis

\section{INTRODUCTION}

Dental implantation has become a principal, established therapy to restore missing natural teeth in regular clinical practice. The rehabilitation technique based on dental implantation can provide a wide variety of treatment options to patients given its high predictability and survival rate, but implants are not completely free of complications and failure. Marginal bone loss (MBL) around dental implants is a serious problem, ${ }^{1-2}$ and extensive bone loss has long been regarded as one key factor contributing to implant failure. ${ }^{3-4}$ Since the 1980 s, MBL assessment with intra-oral radiographs has been regarded as a critical criterion to assess implant success. ${ }^{5}$ The accepted criteria for implant success are defined as $1-1.5 \mathrm{~mm}$ of bone loss during the first year after loading and $<0.2 \mathrm{~mm}$ annually thereafter. ${ }^{6-7}$

MBL, which occurs during the bone-healing period for two-stage implants, exists around non-submerged dental implants and may represent a significant threat to implant longevity. According to the literature, both biological and biomechanical factors may be related to
MBL during bone healing. Host-related factors include plaque control, ${ }^{8}$ smoking ${ }^{9}$ and wound-healing capacity. ${ }^{10-11}$ Implant design characteristics related to MBL may involve platform switching, ${ }^{12}$ the implant surface ${ }^{13}$ and neck microthreads. ${ }^{14}$ Furthermore, other contributing factors, such as surgical traum $\mathrm{a}^{15}$ and different restorative protocols, ${ }^{16}$ may also play a role in this process.

Microbiological studies have demonstrated that the biofilm associated with peri-implantitis or implant failure differs substantially from that associated with healthy implants. ${ }^{17-19}$ Furthermore, interactions among physicochemical surfaces, bacteria and the host immune system are important aspects for determining peri-implant bone loss and the long-term stability of an implant. ${ }^{20}$ These findings inspired the further exploration of the association between oral bacteria and MBL during the bone-healing period. Only limited studies have focused on this relationship.

Notably, almost all previous studies focused on the microenvironment around implants. However, the mouth is an open system with

\footnotetext{
${ }^{1}$ State Key Laboratory of Oral Diseases, National Clinical Research Center for Oral Diseases, West China Hospital of Stomatology, Sichuan University, Chengdu, China; ${ }^{2}$ School of Dentistry, University of California, Los Angeles, CA, USA; ${ }^{3}$ Department of Operative Dentistry and Endodontics, West China Hospital of Stomatology, Sichuan University, Chengdu, China and ${ }^{4}$ Department of Oral Implantology, West China Hospital of Stomatology, Sichuan University, Chengdu, China

Correspondence: Dr Q Yuan, State Key Laboratory of Oral Diseases, National Clinical Research Center for Oral Diseases, West China Hospital of Stomatology, Sichuan University, No. 14, Section 3, Renmin South Road, Chengdu 610041, China

E-mail: yuanquan@scu.edu.cn

Accepted 28 March 2017
} 
continual flow of liquid contacting the surfaces of both hard and soft tissues. Saliva harbours numerous and diverse microorganisms, thus acting as an intermediary for transmitting and dispersing these microorganisms intraorally. ${ }^{21}$ The salivary microbiome has potential connections with the host's health status, and has promise as a surrogate indicator for health monitoring and disease diagnosis. ${ }^{22-23}$ However, no comprehensive study has focused on the correlation between MBL during the bone-healing period and the salivary microbiome, to the best of our knowledge.

Hence, in this study, we employed Illumina MiSeq sequencing to investigate the salivary microbiome associated with MBL before stageII implant surgery. All subjects were divided into three groups (normal, moderate and severe) according to their MBL severities. The working hypothesis was that overall structures and compositions of the three salivary communities are highly correlated with key differences. We hypothesised that individuals with some specific differences in the salivary microbiota may be more prone to MBL during bone healing and that bacteria are significantly associated with MBL progression.

\section{MATERIALS AND METHODS}

\section{Ethics statement}

Participants provided informed consent via a signed statement before participation. The human subject protocol was approved by the Institution Review Board of the West China Hospital of Stomatology, Sichuan University (authorisation number WCHSIRB-ST-2016-072).

\section{Study population}

Participants were recruited from individuals seeking care at the Implant Center of West China Hospital of Stomatology. The following inclusion criteria were used: Chinese, $>18$ years of age, non-smokers in good general healthy, single tissue-level implant with an sandblasted large-grit acid-etched surface (Straumann AG, Basel, Switzerland) in the posterior mandible jaw, and edentulous area and adjacent teeth with healthy periodontal status. The following exclusion criteria were used: antibiotic therapy or oral prophylactic procedures within the preceding 3 months, a need for antibiotic coverage before dental treatment, fewer than 20 teeth present in the dentition; immediate implant or early implant placement, and a need for osseous grafting or other augmentation procedures. All surgical procedures followed the manufacturer's guidelines of Straumann tissue-level implants. All surgical personnel involved in the treatment of these patients had adequate training in advanced implantology.

\section{Clinical examination}

All subjects were examined by one trained and calibrated examiner preoperatively and 3 months postoperatively $\left(\mathrm{T}_{0}\right.$ and $\mathrm{T}_{3}$ ). Plaque indices and the gingival index were measured at four sites per tooth (mesio-buccal, buccal, disto-buccal and lingual). Probe depth ( $\mathrm{mm}$ ) was measured at six sites per teeth (mesio-buccal, buccal, disto-buccal, disto-lingual, lingual and mesio-lingual) using a standard periodontal probe.

\section{Marginal bone-level measurement}

Patients were examined with the same cone beam computed tomography device (3D Accuitomo 170 cone beam computed tomography (CBCT) device, J Morita, Tokyo, Japan) immediately after surgery and 3 months postoperatively. MBL was measured as follows: the implant platform (the horizontal interface between the implant and the abutment) was used as the reference point. Vertical distances from the reference point to the most coronal level of bone- to-implant contact at both the mesial and distal sites were measured at two time points, respectively; ${ }^{24-25} \mathrm{MBL}$ was measured by subtracting the obtained data from each site. The higher value was used to group the patients. Analyses of radiographs were performed by the same investigator who was blinded and unrelated to the study, and all the evaluations were repeated on a separate occasion within a 6-week interval.

\section{Sample collection and DNA isolation}

Saliva was collected before surgery according to the techniques described by Navazesh. ${ }^{26}$ Briefly, $3 \sim 5 \mathrm{~mL}$ spontaneous, whole unstimulated saliva was collected from each subject. Volunteers were instructed to refrain from drinking and eating for at least $2 \mathrm{~h}$ before sampling and from using oral hygiene products for $12 \mathrm{~h}$ before sampling. All samples were stored at $-80^{\circ} \mathrm{C}$ before further processing. Total DNA was extracted using the QIAamp DNA micro Kit (QIAGEN Sciences, MD, USA) with an extra lysozyme treatment step for lysing the bacterial cells.

\section{Sequencing and data analysis}

The V4 regions of the bacterial $16 \mathrm{~S}$ rRNA gene were amplified using polymerase chain reaction $\left(95^{\circ} \mathrm{C}\right.$ for $3 \mathrm{~min} ; 27$ cycles at $95^{\circ} \mathrm{C}$ for $30 \mathrm{~s}$, $55^{\circ} \mathrm{C}$ for $30 \mathrm{~s}, 72^{\circ} \mathrm{C}$ for $45 \mathrm{~s}$; and a final extension at $72{ }^{\circ} \mathrm{C}$ for $10 \mathrm{~min}$ ). The primers 515F $5^{\prime}$-GTGCCAGCMGCCGCGG-3' and 907R 5'-CCGTCAATTCMTTTRAGTTT-3' were used. Purified amplicons were pooled in equimolar ratios and paired-end sequenced $(2 \times 250)$ on an Illumina MiSeq platform (Illumina, San Diego, CA, USA) according to standard protocols. The raw reads were deposited into the NCBI sequence read archive database. Raw fastq files were demultiplexed and quality-filtered using QIIME (version 1.9.1). Operational taxonomical units (OTUs) were clustered with 97\% similarity cutoff using UPARSE (version 7.1), and chimeric sequences were identified and removed using chimaera checking (UCHIME). The taxonomy of each 16S rRNA gene sequence was analysed by RDP Classifier (http://rdp.cme.msu.edu/) against the Silva (SSU123) $16 \mathrm{~S}$ rRNA database using confidence threshold of 70\%. ${ }^{27}$ Alpha-diversity indices were estimated from the number of observed OTUs, Chao1 and the Shannon diversity index, which reveals both species richness and evenness. Non-metric multidimensional scaling (NMDS) ordination was used to determine the degree of dissimilarity between pairs of bacterial communities using the Bray-Curtis distance method. Two different nonparametric analyses were also employed to examine community differences, including analysis of similarities (ANOSIM) and nonparametric multivariate analysis of variance (adonis).

\section{Statistical analysis}

Participants were grouped into the following three categories according to the degree of MBL found around their dental implants: (i) severe group $(n=36): \mathrm{MBL} \geq 1 \mathrm{~mm}$; (ii) moderate group ( $n=36): 0.5 \mathrm{~mm} \leq \mathrm{MBL}<1 \mathrm{~mm}$; and (iii) normal group $(n=28)$ : $\mathrm{MBL}<0.5 \mathrm{~mm}$. The relative abundances of each bacterial taxon were calculated and are typically presented as the mean \pm standard error of the mean (s.e.m.). For multiple comparisons between the three different groups, ANOVA (one-way) and post hoc least significant difference (LSD) was performed. The significance threshold was set at 0.05 .

\section{RESULTS}

Patients' clinical characteristics and overall sequence statistics One hundred volunteers were selected for this study (50 men and 50 women; mean age: 46.78 years; range: 18-60 years; all of Han 
nationality). Clinical metrics for the participants are detailed in Table 1. No significant differences in patients' characteristics were observed among the three groups and between the two time points. In addition, no changes were reported in terms of living habits or health conditions during the bone-healing period.

Sequencing produced a total of 4356945 raw 16S rRNA sequences with an average length of $395 \mathrm{bp}$. After preprocessing, 28 known phyla and 489 genera were identified. A total of 994 OTUs were detected at $3 \%$ dissimilarity using the Uclust programme. ${ }^{28}$ In total, 220 of these were singletons and were excluded from further statistical analysis.

\section{Comparison of the phylogenetic composition among the three salivary communities}

At the phylum level, the microbial compositions of the three communities were similar (Table 2), and the vast majority of sequences $(>95 \%)$ belonged to one of five phyla: Firmicutes, Proteobacteria, Bacteroidetes, Actinobacteria and Fusobacteria. The phyla Spirochaeta $(P<0.05)$ and Synergistetes $(P<0.05)$ were highly associated with the moderate and severe groups, and TM7 (Candidatus Saccharibacteria) and Tenericutes were also more abundant in those two groups than in the normal group. Among all the three groups, the five predominant phyla were present at similar levels.
We then investigated the microbial shift in more detail at the genus level. A genus-level phylogenetic tree constructed using MEGA 5 is presented in Supplementary Figure S1. The overall abundance and the magnitude of the difference across the normal, moderate and severe groups are indicated by the bars. Streptococcus, Lautropia, Neisseria, Oribacterium, Actinomyces, Prevotella [G-7] and Selenomonas [G-3] were the predominant genera. With the exception of the genera of Actinomyces, Prevotella [G-7] and Selenomonas [G-3], the abundances of which were slightly altered, the top seven genera remained almost the same as severity increased from normal to severe. Figure 1 presents genera for which the abundance differed by $\geq 0.1 \%$ between the groups. Figure 2 presents the genera for which the abundance significantly differed among the three groups. Compared with the normal group, the relative abundance of species in the genus Treponema was significantly increased $(P<0.05)$ in the moderate group. Neisseria, Oribacterium, Selenomonas [G-3] and Capnocytophaga were more abundant in the normal group than in the severe group. In contrast, the reverse trend was noted for Haemophilus, Leptotrichia and Veillonella, which were significantly $(P<0.05)$ more abundant in the normal group.

The differences in bacterial composition among the three groups were also reflected by the Venn diagram of shared and different OTUs (Supplementary Figure S2). In total, 54.9\% of the total OTUs detected

Table 1 Demographics and clinical parameters of all subjects

\begin{tabular}{|c|c|c|c|c|c|c|}
\hline \multirow[b]{3}{*}{ Characteristics } & \multicolumn{6}{|c|}{ Groups } \\
\hline & \multicolumn{2}{|c|}{ Normal $(n=28)$} & \multicolumn{2}{|c|}{ Moderate $(n=36)$} & \multicolumn{2}{|c|}{ Severe $(n=36)$} \\
\hline & $\mathrm{T}_{0}$ & $\mathrm{~T}_{3}$ & $\mathrm{~T}_{0}$ & $\mathrm{~T}_{3}$ & $\mathrm{~T}_{0}$ & $\mathrm{~T}_{3}$ \\
\hline Male/Female & $12 / 16$ & - & $16 / 20$ & - & $18 / 18$ & - \\
\hline Age (mean \pm s.d.) & $42.0 \pm 14.6$ & - & $45.0 \pm 14.1$ & - & $52.3 \pm 15.9$ & - \\
\hline Alcohol drinking & 0 & 0 & 0 & 0 & 0 & 0 \\
\hline Number of teeth (mean \pm s.d.) & $28.05 \pm 2.25$ & - & $28.42 \pm 1.77$ & - & $27.34 \pm 2.58$ & - \\
\hline Plaque index (mean \pm s.d.) & $1.21 \pm 0.61$ & $1.20 \pm 0.60$ & $1.39 \pm 0.60$ & $1.39 \pm 0.54$ & $1.44 \pm 0.69$ & $1.42 \pm 0.68$ \\
\hline Gingival index (mean \pm s.d.) & $1.08 \pm 0.54$ & $1.06 \pm 0.52$ & $1.28 \pm 0.50$ & $1.24 \pm 0.51$ & $1.40 \pm 0.50$ & $1.35 \pm 0.54$ \\
\hline Probing depth, mm (mean \pm s.d.) & $2.02 \pm 0.73$ & $2.01 \pm 0.69$ & $2.25 \pm 0.60$ & $2.20 \pm 0.54$ & $2.51 \pm 1.44$ & $2.33 \pm 1.39$ \\
\hline $\mathrm{MBL}, \mathrm{mm}$ (mean \pm s.d.) & \multicolumn{2}{|c|}{$0.28 \pm 0.13$} & \multicolumn{2}{|c|}{$0.71 \pm 0.13$} & \multicolumn{2}{|c|}{$1.55 \pm 0.53$} \\
\hline
\end{tabular}

$\mathrm{T}_{0}$ : baseline (recruitment).

$\mathrm{T}_{3}$ : 3 months after implant surgery.

s.d., standard deviation.

Table 2 The relative abundance (mean \pm s.e.m.) of the bacterial phylum among the normal, moderate and severe groups

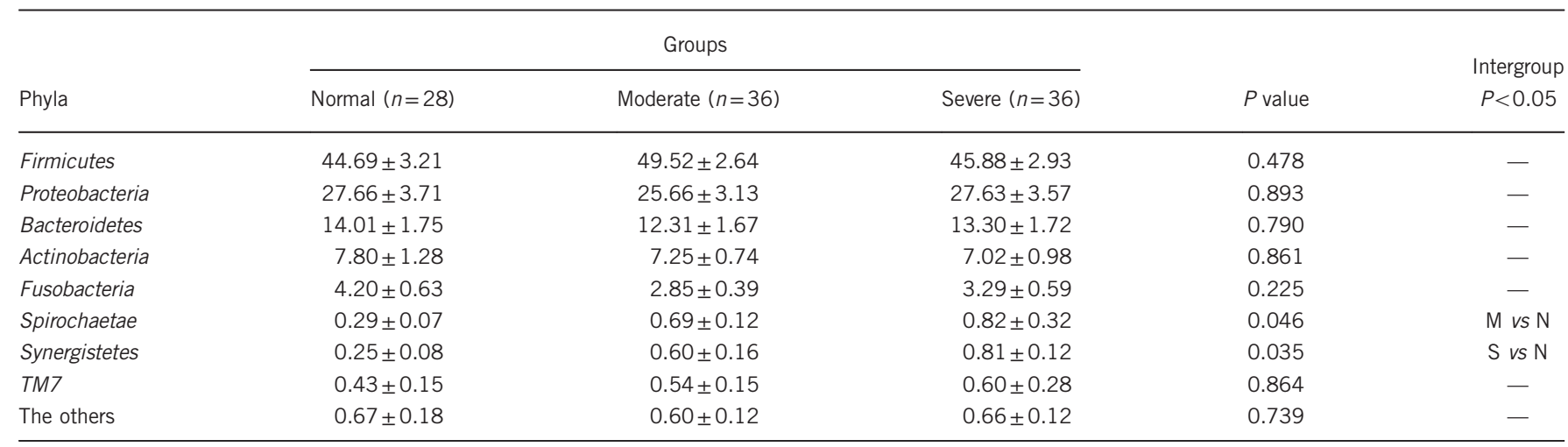

$\mathrm{M}$, moderate group; $\mathrm{N}$, normal group; $\mathrm{S}$, severe group; s.e.m., standard error of the mean.

ANOVA (one-way) and post hoc least significant difference (LSD) were performed for multiple comparisons between the three different niches. The significance threshold was set at 0.05 . 


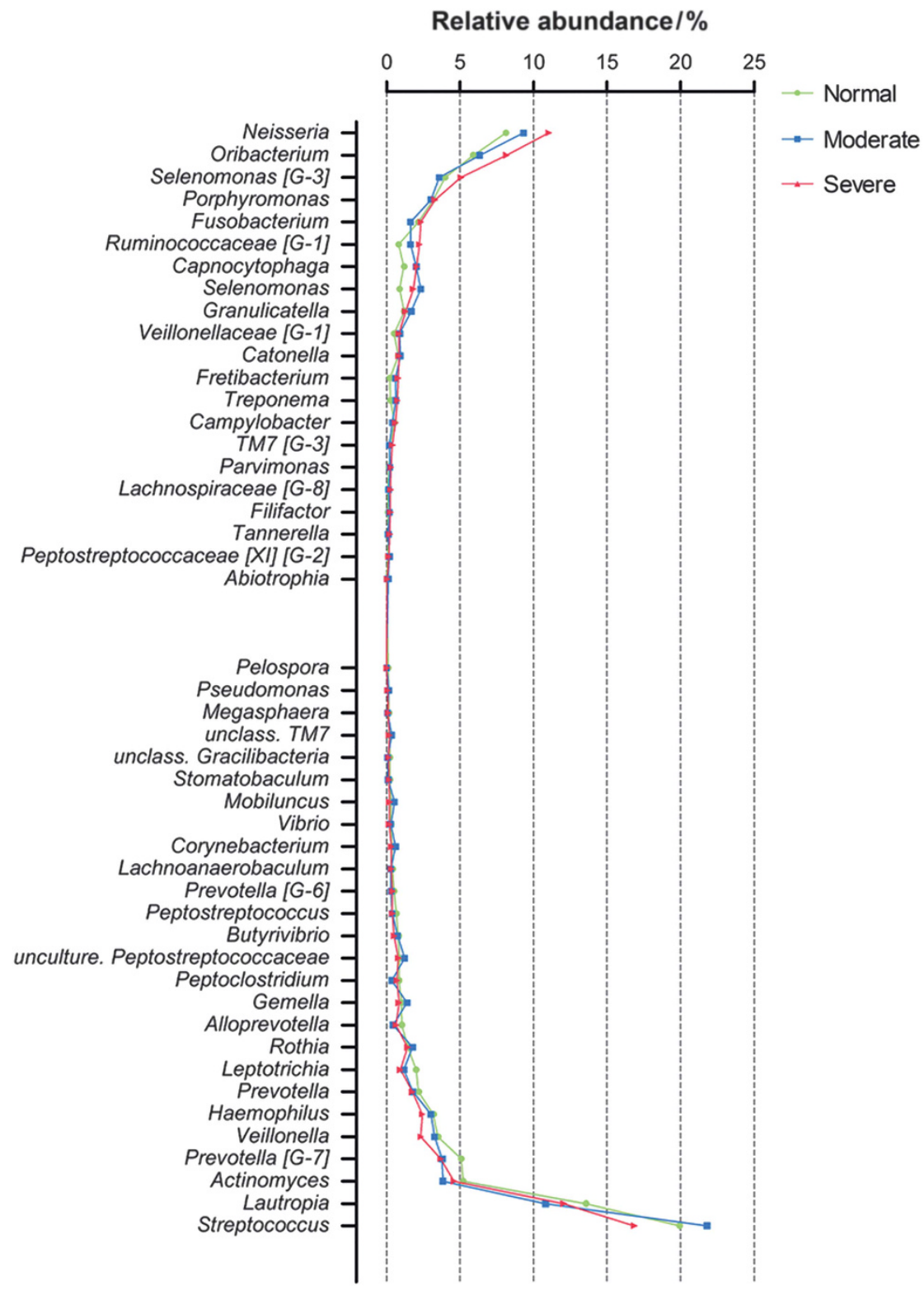

Figure 1 Microbial differences among the normal, moderate and severe groups at the genus level. The graph presents levels for the genera for which the abundances were $\geq 0.1 \%$ different between groups. The taxa were sorted according to the magnitude of change.

(774, with the exception of singletons) were shared among all three communities. The numbers of OTUs exclusively detected in the severe, moderate and normal groups were 54,66 and 35 , respectively. Twenty-two OTUs were shared between the severe and normal groups, and 27 OTUs were shared between the moderate and normal groups. However, more OTUs (66) were shared between the moderate and severe groups.

In addition, we evaluated the OTUs that were present in two or more samples with a mean relative abundance of $>0.01 \%$ (Supplementary Table S2). In total, 29 of the 250 health-associated OTUs were significantly different among the three groups $(P<0.05)$. Notably, most of those OTUs demonstrating statistically increased abundances in the severe group were Gram-negative anaerobic taxa.
Comparison of the phylogenetic structure among the three salivary communities

We visualised the differences in the phylogenetic structures among the groups by performing a NMDS ordination. Each data point represents one sample, and the spatial distance between points in the plot is interpreted as the relative difference in the composition of substrate marking. NMDS for these taxa failed to indicate an obvious separation among normal, moderate and severe subjects. The results reveal slight tight clustering of normal samples, but a broader variation in the moderate and severe samples (Figure 3). This difference was more obvious between the severe and normal groups than between the moderate and normal groups. In addition, dissimilarity tests (Adonis and ANOSIM) among the groups also revealed the same trend; however, the differences were not statistically significant $(P>0.05$, Supplementary Table S1). We also 

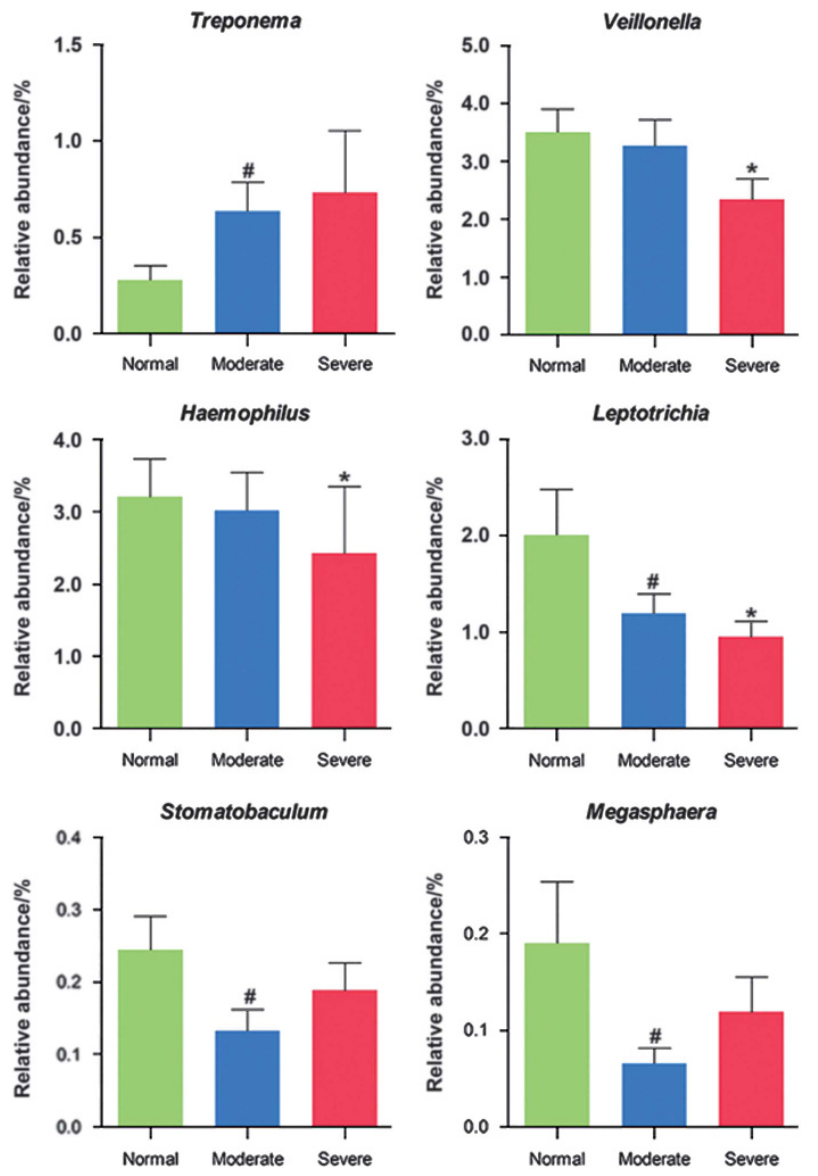

Figure 2 Genera for which the abundances significantly differed between the groups. ANOVA (one-way) and post hoc least significant difference (LSD) were performed for multiple comparisons between the three different groups. ${ }^{*} P<0.05$ between severe group and normal group; ${ }^{P} P<0.05$ between moderate group and normal group.

examined the differences using the observed OTUs, the Chao index and the Shannon index, and found no significant trends. Oral microbial diversity and richness were similar among the three groups (Supplementary Figure S3).

Changes in the core microbiota across each community

We examined the core microbiota communities found in periimplantitis as identified in previous studies. ${ }^{29-30} \mathrm{We}$ investigated the most abundant species ( $>0.5 \%$ abundance) among the various communities (Figure 4). Among the total of 41 species, 28 were common to three communities. The numbers of species unique to each community from the normal, moderate and severe groups were three, two and four, respectively. Three species (Neisseria mucosa, Capnocytophaga leadbetteri and Fretibacterium sp. IHOT_359|) were identified in both the moderate and severe groups but not in the normal group. One species was shared by the normal and moderate groups. However, no species were shared by the normal and severe groups. These results indicated that although the three communities had the similar overall phylogenetic compositions, some specific species differed significantly during the shift from the normal setting to a disease state (Figure 5).
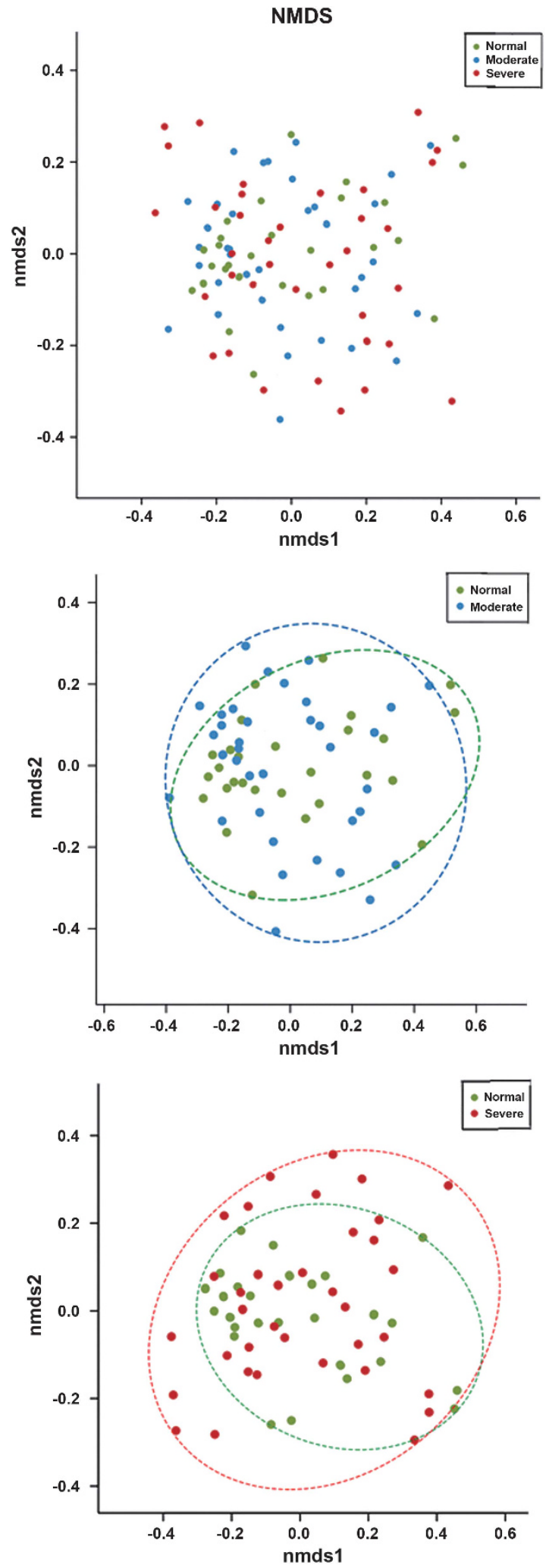

Figure 3 NMDS analysis. NMDS based on the Bray-Curtis distance among all the three groups, and between the normal group and moderate or severe groups. 


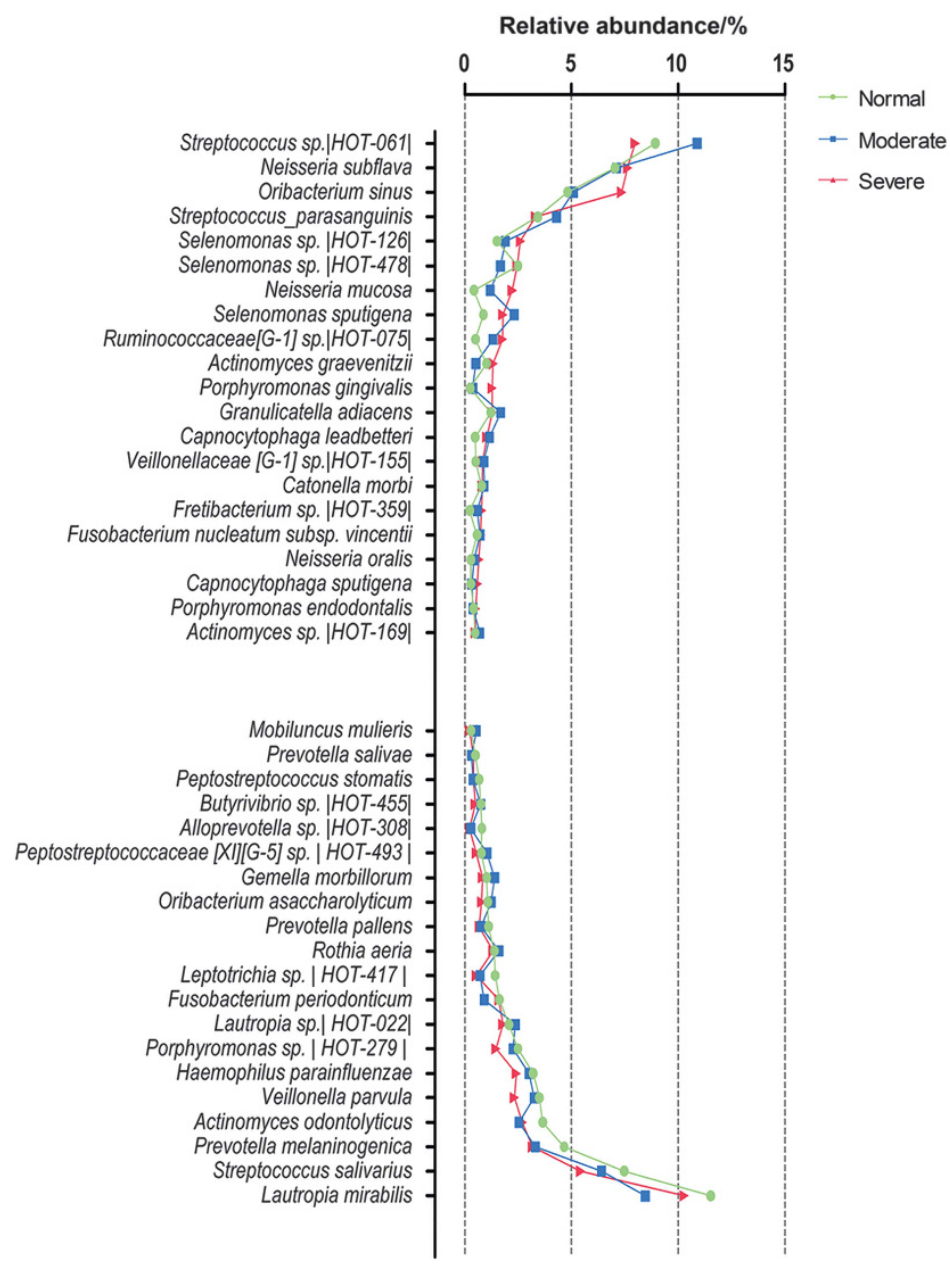

Figure 4 Microbial differences among the normal, moderate and severe groups at the species level. The graph presents the most abundant species $(\geq 0.5 \%$ abundance) in the normal, moderate and severe samples. The taxa were sorted according to the magnitude of change. The species name or human oral taxon ID in the human oral microbiome is presented.
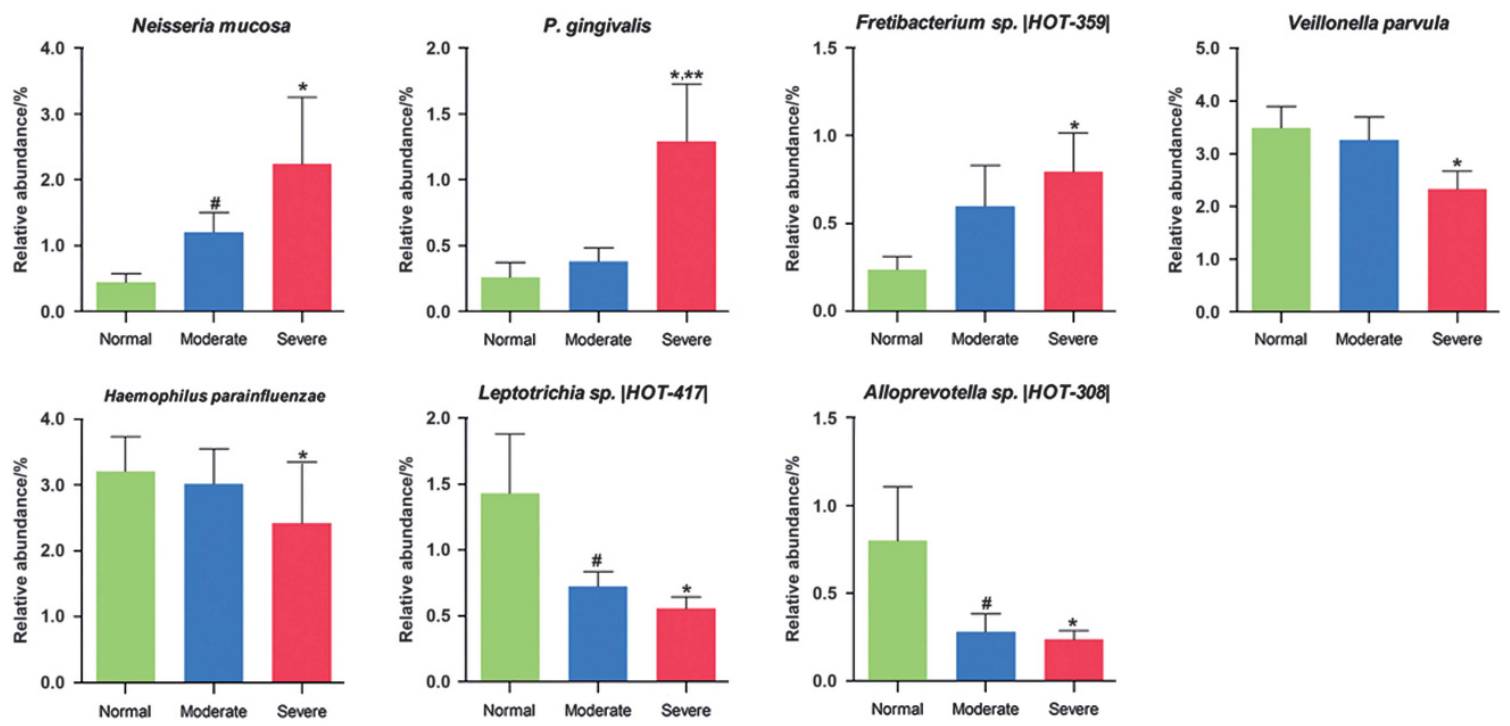

Figure 5 Species for which the abundances significantly differed between the groups. ANOVA (one-way) and post hoc least significant difference (LSD) were performed for multiple comparisons between the three different groups. ${ }^{*} P<0.05$ between severe group and normal group; ${ }^{*} P<0.05$ between severe group and moderate group; ${ }^{\#}<0.05$ between moderate group and normal group. 
Table 3 The relative abundance (mean \pm s.e.m.) of the species associated with disease among the normal, moderate and severe groups

\begin{tabular}{|c|c|c|c|c|c|}
\hline \multirow[b]{2}{*}{ Species } & \multicolumn{3}{|c|}{ Groups } & \multirow[b]{2}{*}{$P$ value } & \multirow{2}{*}{$\begin{array}{c}\text { Intergroup } \\
P<0.05\end{array}$} \\
\hline & Normal $(n=28)$ & Moderate $(n=36)$ & Severe $(n=36)$ & & \\
\hline & \multicolumn{4}{|c|}{ Red complex } & \\
\hline Porphyromonas gingivali & $0.25 \pm 0.12$ & $0.38 \pm 0.11$ & $1.29 \pm 0.43$ & 0.020 & $\mathrm{~S}$ vs $\mathrm{N} ; \mathrm{S}$ vs $\mathrm{M}$ \\
\hline Tannerella forsythia & $0.13 \pm 0.04$ & $0.12 \pm 0.02$ & $0.20 \pm 0.04$ & 0.223 & - \\
\hline Treponema denticola & $0.03 \pm 0.01$ & $0.05 \pm 0.01$ & $0.09 \pm 0.01$ & 0.046 & $\mathrm{M}$ vs $\mathrm{N}$ \\
\hline Eubacterium nodatum & $0.03 \pm 0.01$ & $0.07 \pm 0.02$ & $0.07 \pm 0.03$ & 0.349 & - \\
\hline \multirow[t]{2}{*}{ Treponema socranskii } & $0.03 \pm 0.01$ & $0.06 \pm 0.02$ & $0.09 \pm 0.05$ & 0.441 & - \\
\hline & \multicolumn{4}{|c|}{ Orange complex } & \\
\hline Prevotella melaninogenica & $4.65 \pm 0.90$ & $3.31 \pm 0.84$ & $3.18 \pm 0.76$ & 0.420 & - \\
\hline Prevotella intermedia & $0.41 \pm 0.10$ & $0.34 \pm 0.09$ & $0.47 \pm 0.16$ & 0.757 & - \\
\hline Prevotella nigrescens & $0.05 \pm 0.02$ & $0.06 \pm 0.02$ & $0.05 \pm 0.03$ & 0.969 & - \\
\hline Fusobacterium nucleatum subsp. vincentii & $0.57 \pm 0.12$ & $0.72 \pm 0.15$ & $0.74 \pm 0.15$ & 0.688 & - \\
\hline \multirow[t]{2}{*}{ Fusobacterium periodonticum } & $1.62 \pm 0.29$ & $0.92 \pm 0.16$ & $1.60 \pm 0.40$ & 0.164 & - \\
\hline & \multicolumn{4}{|c|}{ Yellow complex } & \\
\hline Streptococcus sanguinis & $1.42 \pm 0.28$ & $1.97 \pm 0.39$ & $1.57 \pm 0.43$ & 0.593 & - \\
\hline Streptococcus intermedius & $0.12 \pm 0.03$ & $0.28 \pm 0.06$ & $0.42 \pm 0.07$ & 0.045 & $\mathrm{~S}$ vs $\mathrm{N} ; \mathrm{M}$ vs $\mathrm{N}$ \\
\hline
\end{tabular}

$\mathrm{M}$, moderate group; $\mathrm{N}$, normal group; S, severe group; s.e.m., standard error of the mean.

ANOVA (one-way) and post hoc least significant difference (LSD) were performed for multiple comparisons between the three different groups. The significance threshold was set at 0.05 .

\section{Species associated with disease}

Considering that MBL was caused by intra-individual microbial infections, we expected that we would observe species that were common to MBL in periodontitis and peri-implantitis. We evaluated the species that are the important periodontal pathogens belonging to the "red complex", "orange complex" and "yellow complex", as well as the species having closely relationship with these complexes ${ }^{31-32}$ (Table 3$)$. We found that $P$. gingivalis $(P<0.05)$ and Treponemadenticola $(P<0.05)$, which are important periodontal pathogens of the "red complex", were abundant and prevalent in the moderate and severe groups. The moderate and severe groups also appeared to have higher levels of Streptococcus intermedius $(P<0.05)$, which belongs to the "yellow complex", than did the normal group.

\section{DISCUSSION}

Research on peri-implant diseases has primarily focused on the late disease stages. However, peri-implant tissue destruction sometimes occurs, and little is known about the how this process is initiated. We are aware of factors that influence MBL during bone healing, but there is a paucity of knowledge regarding the role of bacteria in the progression of this common complication. In this pilot study, we first investigated the complexity of oral microbial communities in patients with different levels of MBL around dental implant during bone healing using MiSeq sequencing. Our results demonstrated the differences between communities at three levels of MBL severity and the correlations of these communities with MBL during bone healing.

Saliva is formed by the mixing of liquid products of the salivary glands, including components of gingival crevicular fluid, serum, bacteria and their products, viruses, fungi, peeled epithelial cells and food particles. Saliva is responsible for maintaining the integrity of the oral cavity. Over the past decade, a number of studies have used saliva samples as an easy, inexpensive and non-invasive diagnostic tool to assess healthy and disease conditions. ${ }^{33-35}$ Saliva can be considered as a mirror of body health given its association with various oral and systemic conditions, including caries, cardiovascular disease and obesity. ${ }^{36-37}$ The salivary microbiome, which is specific to each person, exhibits long-term stability on the scale of years. ${ }^{38}$ Inspired by these findings, we collected saliva samples from 100 individuals who were divided into three categories on the basis of MBL severity.

NMDS analysis revealed a tendency for clustering with a narrower distribution of samples in the normal group. Dissimilarity tests revealed " $\mathrm{S} v s \mathrm{~N}$ " had the highest $R$-value among the three comparisons. These results indicate that the difference between the severe and normal groups was more obvious than that between moderate and normal groups. This finding may indicate that MBL during bone healing is a complication associated with endogenous bacteria. Rather than being caused by specific pathogens, it is more likely caused by an alteration in the densities of commensal oral bacteria.

Among the three groups, five major phyla constituted the predominant salivary microbiome (namely, Firmicutes, Proteobacteria, Bacteroidetes, Actinobacteria and Fusobacteria). This finding is consistent with a previous survey on the "core microbiome" of the oral microbial community. ${ }^{39}$ Significant differences were identified among the three groups. The relative abundances of the phyla Spirochaeta and Synergistetes increased as MBL severity increased. A similar trend was noted for the phylum $T M 7$, although it was not statistically significant. Both Spirochaeta and Synergistetes are Gram-negative anaerobic taxa. Salivary glycoproteins selectively adhere to the abutment or other surfaces of an implant to form the salivary pellicle, where oral bacteria then attach by adhering to epitopes in the pellicle. ${ }^{40}$ In general, the primary colonisers tend to be Gram-positive aerobes and facultative anaerobes, ${ }^{41}$ whereas Gram-negative and anaerobic species are found at increased abundances in mature plaques. ${ }^{42}$ Bone loss around an implant can be stimulated by bacteria, and bone loss subsequently stimulates an anaerobic environment. Together, these conditions 
become the primary cause of continued bone loss. ${ }^{43}$ Early studies have demonstrated that the subgingival biofilms around failing implants have similar compositions that are characterised by a high proportion of Gram-negative anaerobic rods. ${ }^{29,44}$ The increased abundances of species in these phyla were in consistent with the previous studies that reported evidence that they were newly identified taxa associated with periodontitis. ${ }^{45-48}$ The phylum TM7 also exhibits an association with peri-implant diseases. ${ }^{30}$

Increased MBL was positively related at the genus level to the proportion of the genus Treponema that belongs to the phylum Spirochaeta. The normal group demonstrated increases in the relative abundances of species in the genera Veillonella, Haemophilus and Leptotrichia. Veillonella species interact with Streptococcus species during biofilm formation ${ }^{49}$ and thus are likely associated with implant health. Nevertheless, Griffen et al. ${ }^{30}$ suggested that Veillonella had a positive association with periodontitis. Haemophilus species are Gramnegative facultative bacilli, and $H$. influenza is detected in higher amounts in peri-implant lesions. ${ }^{50-51}$ In the present study, this bacterium was not identified in any of the samples. Many of the species present were unclassified taxa except Haemophilus parainfluenzae. Further work is needed to investigate the association between Haemophilus species and destructive peri-implant infection. A previous study also reported a similar trend, showing that the periimplantitis-associated community had a significantly lower level of the genus Leptotrichia. ${ }^{19}$ In addition, the abundance of the Gram-positive facultative coccus Actinomyces decreased as severity increased, which is consistent with the findings of a previous study. ${ }^{52}$

Notably, we found that $P$. gingivalis and $T$. denticola were significantly abundant in the moderate and/or severe groups. The proportions of the pathogens from the red complex were elevated as severity increased. Members of red complex of periodontal pathogens were previously demonstrated to be the most important microbiota for the progression of periodontitis ${ }^{31}$ and to be positively associated with peri-implantitis. ${ }^{17,29,51}$ The relative abundance of $P$. gingivalis, a proposed keystone organism in chronic periodontitis, increased significantly as the severity increased from normal to severe. In addition, its prevalence rates also increased consistently (71\%, 75\% and $92 \%$, respectively). These results indicate that $P$. gingivalis might act as a predictor of MBL. Furthermore, $P$. gingivalis manipulates immunity via a number of mechanisms, ${ }^{53}$ and has been postulated to suppress inflammasome activation by both its immunostimulatory activity and pathogenic synergy with other periodontal bacteria. ${ }^{54}$ Recent studies have also revealed its relationship with systemic and oral health. ${ }^{55-56}$

The abundance of Streptococcus intermedius, which belongs to the yellow complex, exhibited a significantly positive correlation with the severity of MBL during bone healing. This finding is consistent with previous studies suggesting that it is associated with periodontitis. ${ }^{48,57}$ Streptococcus sanguinis, which also belongs to the yellow complex, exhibited a slightly increased abundance in the moderate and severe groups. S. sanguinis is a primary coloniser of oral plaques and has been found to be associated with dental implants. ${ }^{58-59}$

A very interesting finding is that Neisseria mucosa was only found in the moderate and severe groups when analysed at the level of most abundant species. N. mucosa is a pathogenic bacterium that is a rare but serious cause of endocarditis. ${ }^{60}$ The correlation between it with MBL merits future exploration.

To investigate the relationship between MBL during bone healing and the salivary microbiome in a clinical setting, we recruited patients who exhibited no significant differences in clinical metrics among the three groups at baseline and 3 months after surgery. In addition, no significant differences were noted between the two time points. We also controlled other possible factors that may influence MBL: smoking habits, systemic health and characteristics of the implants. ${ }^{61-62}$ However, this present study is only a pilot study to investigate the correlation between the MBL during bone healing and the salivary microbiome, and many interactions between species or between species and oral health remain unknown. Further studies are needed to determine the roles of species that are associated with oral health. To shed more light on clinical mechanisms and disease progression, prospectively designed studies that rigorously control confounding factors and have long-term follow-ups (e.g., 1 year after implant surgery), and multicentre clinical trials, are required in the future.

In conclusion, the current study demonstrates that the severity of peri-implant MBL during bone healing is correlated with different salivary microbiomes. This finding suggests a role for the oral microbiota in the onset/progression of MBL. Our findings could provide the basis for further work exploring the bacteria suspected of being associated with MBL in the future. The identification of specific bacterial species that are involved in the progression of peri-implantitis could also provide potential targets for a better prevention programme for MBL during the bone-healing period.

\section{ACKNOWLEDGEMENTS}

This work was supported by grants from the National Natural Science Foundation of China (NSFC81371173; 81571001), State Key Laboratory of Oral Diseases (SKLOD201704), and International Team for Implantology (Grant No. 975_2014, Basel, Switzerland) to Quan Yuan; the National Key R \& D Program of China during the 13th Five-Year Plan (2016YFC1102700) to XueDong Zhou. The funders had no role in study design, data collection and analysis, decision to publish, or preparation of the manuscript. The authors have stated explicitly that there are no conflict of interest in connection with this article.

1 Roos-Jansåker AM, Lindahl C, Renvert $\mathrm{H}$ et al. Nine- to fourteen-year follow-up of implant treatment. Part II: presence of peri-implant lesions. J Clin Periodontol 2006; 33(4): 290-295.

2 Fransson C, Lekholm U, Jemt T et al. Prevalence of subjects with progressive bone loss at implants. Clin Oral Implants Res 2005; 16(4): 440-446.

3 Esposito M, Hirsch JM, Lekholm U et al. Biological factors contributing to failures of osseointegrated oral implants. (I). Success criteria and epidemiology. Eur J Oral Sci 1998; 106(1): 527-551.

4 Esposito M, Hirsch JM, Lekholm U et al. Biological factors contributing to failures of osseointegrated oral implants. (II). Etiopathogenesis. Eur J Oral Sci 1998; 106(3): 721-764.

5 Albrektsson T, Zarb G, Worthington P et al. The long-term efficacy of currently used dental implants: a review and proposed criteria of success. Int J Oral Maxillofac Implants 1986; 1(1): 11-25.

6 Smith DE, Zarb GA. Criteria for success of osseointegrated endosseous implants. J Prosthet Dent 1989; 62(5): 567-572.

7 Misch CE, Perel ML, Wang HL et al. Implant success, survival, and failure: the International Congress of Oral Implantologists (ICOI) Pisa Consensus Conference. Implant Dent 2008; 17(1): 5-15.

$8 \mathrm{Kim}$ TH, Lee DW, Kim CK et al. Influence of early cover screw exposure on crestal bone loss around implants: intraindividual comparison of bone level at exposed and nonexposed implants. J Periodontol 2009; 80(6): 933-939.

9 Shibli JA, Piattelli A, lezzi $G$ et al. Effect of smoking on early bone healing around oxidized surfaces: a prospective, controlled study in human Jaws. J Periodontol 2010; 81(4): 575-583.

10 Oates TW, Dowell S, Robinson M et al. Glycemic control and implant stabilization in type 2 diabetes mellitus. J Dent Res 2009; 88(4): 367-371.

11 Javed F, Romanos GE. Impact of diabetes mellitus and glycemic control on the osseointegration of dental implants: a systematic literature review. J Periodontol 2009; 80(11): 1719-1730.

12 Becker J, Ferrari D, Mihatovic I et al. Stability of crestal bone level at platform-switched non-submerged titanium implants: a histomorphometrical study in dogs. J Clin Periodontol 2009; 36(6): 532-539. 
13 Frenkel SR, Simon J, Alexander $\mathrm{H}$ et al. Osseointegration on metallic implant surfaces: effects of microgeometry and growth factor treatment. J Biomed Mater Res 2002; 63(6): 706-713

14 Mangano C, Mangano F, Piattelli A et al. Prospective clinical evaluation of 1920 Morse taper connection implants: results after 4 years of functional loading. Clin Oral Implants Res 2009; 20(3): 254-261.

15 Blanco J, Nuñez V, Aracil L et al. Ridge alterations following immediate implant placement in the dog: flap versus flapless surgery. J Clin Periodontol 2008; 35(7): 640-648.

16 Ghoveizi R, Alikhasi M, Siadat MR et al. A radiographic comparison of progressive and conventional loading on crestal bone loss and density in single dental implants: a randomized controlled trial study. J Dent 2013; 10(2): 155-163.

17 Shibli JA, Melo L, Ferrari DS et al. Composition of supra- and subgingival biofilm of subjects with healthy and diseased implants. Clin Oral Implants Res 2008; 19(10): 975-982.

18 Renvert S, Roos-Jansaker AM, Lindahl CA et al. Infection at titanium implants with or without a clinical diagnosis of inflammation. Clin Oral Implants Res 2007; 18(4): 509-516.

19 Kumar PS, Mason MR, Brooker MR et al. Pyrosequencing reveals unique microbial signatures associated with healthy and failing dental implants. J Clin Periodontol 2012; 39(5): 425-433.

20 Tabanella G, Nowzari H, Slots J. Clinical and microbiological determinants of ailing dental implants. Clin Implant Dent Relat Res 2009; 11(1): 24-36.

21 Kaplan JB. Biofilm dispersal: mechanisms, clinical implications, and potential therapeutic uses. J Dent Res 2010; 89(3): 205-218.

22 Zhang CZ, Cheng XQ, Li JY et al. Saliva in the diagnosis of diseases. Int J Oral Sci 2016; 8(3): 133-137.

23 Yoshizawa JM, Schafer CA, Schafer JJ et al. Salivary biomarkers: toward future clinical and diagnostic utilities. Clin Microbiol Rev 2013; 26(4): 781-791.

24 Zhang XX. Shi JY, Gu YX et al. Long-term outcomes of early loading of straumann implant-supported fixed segmented bridgeworks in edentulous maxillae: a 10-year prospective study. Clin Implant Dent Relat Res 2016; 18(6): 1227-1237.

25 Kumar VV, Sagheb K, Kämmerer PW et al. Retrospective clinical study of marginal bone level changes with two different Screw-Implant types: comparison between tissue level (TE) and bone level (BL) implant. J Maxillofac Oral Surg 2014; 13(3): 259-266.

26 Navazesh M. Methods for collecting saliva. Ann N Y Acad Sci 1993; 694: 72-77.

27 Amato KR, Yeoman CJ, Kent A et al. Habitat degradation impacts black howler monkey (Alouatta pigra) gastrointestinal microbiomes. ISME J 2013; 7(7): 1344-1353.

28 Edgar RC. Search and clustering orders of magnitude faster than BLAST. Bioinformatics 2010; 26(19): 2460-2461.

29 Maruyama N, Maruyama F, Takeuchi Y et al. Intraindividual variation in core microbiota in peri-implantitis and periodontitis. Sci Rep 2014; 4: 6602.

30 Griffen AL, Beall CJ, Campbell JH et al. Distinct and complex bacterial profiles in human periodontitis and health revealed by 16 S pyrosequencing. ISME J 2012; 6(6): 1176-1185.

31 Socransky SS, Haffajee AD, Cugini MA et al. Microbial complexes in subgingival plaque. J Clin Periodontol 1998; 25(2): 134-144.

32 Haffajee AD, Socransky SS, Patel MR et al. Microbial complexes in supragingival plaque. Oral Microbiol Immunol 2008; 23(3): 196-205.

33 Prasad S, Tyagi AK, Aggarwal BB. Detection of inflammatory biomarkers in saliva and urine: potential in diagnosis, prevention, and treatment for chronic diseases. Exp Biol Med 2016; 241(8): 783-799.

34 Khurshid Z, Zohaib S, Najeeb S et al. Advances of proteomic sciences in dentistry. Int J Mol Sci 2016; 17(5): 728.

$35 \mathrm{Ai}$ JY, Smith B, Wong DT. Bioinformatics advances in saliva diagnostics. Int J Oral Sci 2012; 4(2): 85-87.

36 Goodson JM, Groppo D, Halem S et al. Is obesity an oral bacterial disease? J Dent Res 2009; 88(6): 519-523.

37 Crielaard W, Zaura E, Schuller AA et al. Exploring the oral microbiota of children at various developmental stages of their dentition in the relation to their oral health. $B M C$ Med Genomics 2011; 4: 22.

38 Yamanaka W, Takeshita T, Shibata Y et al. Compositional stability of a salivary bacterial population against supragingival microbiota shift following periodontal therapy. PLoS One 2012; 7(8): e42806.

39 Zaura E, Keijser BJ, Huse SM et al. Defining the healthy 'core microbiome' of oral microbial communities. BMC Microbiol 2009; 9: 259.
40 Murray PA, Prakobphol A, Lee T et al. Adherence of oral streptococci to salivary glycoproteins. Infect Immun 1992; 60(1): 31-38.

41 Nyvad B, Kilian M. Microbiology of the early colonization of human enamel and root surfaces in vivo. Scand J Dent Res 1987; 95(5): 369-380.

42 Zijnge V, Van Leeuwen MB, Degener JE et al. Oral biofilm architecture on natural teeth. PLoS One 2010; 5(2): e9321.

43 Mombelli A. Microbiology and antimicrobial therapy of peri-implantitis. Periodontol 2000 2002; 28: 177-189.

44 Botero JE, González AM, Mercado RA et al. Subgingival microbiota in peri-implant mucosa lesions and adjacent teeth in partially edentulous patients. J Periodontol 2005 ; 76(9): 1490-1495.

45 You M, Mo S, Watt RM et al. Prevalence and diversity of Synergistetes taxa in periodontal health and disease. J Periodontal Res 2013; 48(2): 159-168.

46 You M, Mo S, Leung WK et al. Comparative analysis of oral treponemes associated with periodontal health and disease. BMC Infect Dis 2013; 13: 174.

47 Vartoukian SR, Palmer RM, Wade WG. Diversity and morphology of members of the phylum 'synergistetes' in periodontal health and disease. App/ Environ Microbiol 2009, 75(11): 3777-3786

48 Abusleme L, Dupuy AK, Dutzan $\mathrm{N}$ et al. The subgingival microbiome in health and periodontitis and its relationship with community biomass and inflammation. ISME 2013; 7(5): 1016-1025.

49 Mashima I, Nakazawa F. The influence of oral Veillonella species on biofilms formed by Streptococcus species. Anaerobe 2014; 28: 54-61.

50 Shibli JA, Martins MC, Lotufo RF et al. Microbiologic and radiographic analysis of ligature-induced peri-implantitis with different dental implant surfaces. Int J Oral Maxillofac Implants 2003; 18(3): 383-390.

51 Persson GR, Renvert S. Cluster of bacteria associated with peri-implantitis. Clin Implant Dent Relat Res 2014; 16(6): 783-793.

52 Faveri M, Figueiredo LC, Shibli JA et al. Microbiological diversity of peri-implantitis biofilms. Adv Exp Med Biol 2015; 830: 85-96.

53 Hajishengallis G, Lamont RJ. Breaking bad: manipulation of the host response by Porphyromonas gingivalis. Eur J Immunol 2014; 44(2): 328-338.

54 Taxman DJ, Swanson KV, Broglie PM et al. Porphyromonas gingivalis mediates inflammasome repression in polymicrobial cultures through a novel mechanism involving reduced endocytosis. J Biol Chem 2012; 287(39): 32791-32799.

55 Wada K, Kamisaki Y. Roles of oral bacteria in cardiovascular diseases-from molecular mechanisms to clinical cases: Involvement of Porphyromonas gingivalis in the development of human aortic aneurysm. J Pharmacol Sci 2010; 113(2): $115-119$.

56 Amar S, Leeman S. Periodontal innate immune mechanisms relevant to obesity. $\mathrm{Mol}$ Oral Microbiol 2013; 28(5): 331-341.

57 Kumar PS, Griffen AL, Moeschberger ML et al. Identification of candidate periodontal pathogens and beneficial species by quantitative $16 \mathrm{~S}$ clonal analysis. J Clin Microbio 2005; 43(8): 3944-3955.

58 Nascimento CD, Pita MS, Fernandes FH et al. Bacterial adhesion on the titanium and zirconia abutment surfaces. Clin Oral Implants Res 2014; 25(3): 337-343.

59 Fürst MM, Salvi GE, Lang NP et al. Bacterial colonization immediately after installation on oral Titanium implants. Clin Oral Implants Res 2007; 18(4): 501-508.

60 Pilmis B, Lefort A, Lecuit M et al. Endocarditis due to Neisseria mucosa: case report and review of 21 cases: a rare and severe cause of endocarditis. J Infect 2014; 68(6): 601-604.

61 Tatarakis N, Bashutski J, Wang HL et al. Early implant bone loss: preventable or inevitable? Implant Dent 2012; 21(5): 379-386.

62 Oh TJ, Yoon J, Misch CE et al. The causes of early implant bone loss: myth or science? J Periodontol 2002; 73(3): 322-333.

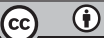

This work is licensed under a Creative Commons Attribution 4.0 International License. The images or other third party material in this article are included in the article's Creative Commons license, unless indicated otherwise in the credit line; if the material is not included under the Creative Commons license, users will need to obtain permission from the license holder to reproduce the material. To view a copy of this license, visit http://creativecommons.org/licenses/by/4.0/

(C) The Author(s) 2017

Supplementary Information for this article can be found on the International Journal of Oral Science website (http://www.nature.com/ijos) 